Received: May 21, 2017

Revision received: January 27, 2018

\title{
Application of MIDI Software and Controller in Music Teaching*
}

\author{
Jiaojing You ${ }^{1}$ \\ Hunan City University
}

\begin{abstract}
MIDI software and controllers are highly practical and modern teaching methods. They are very helpful for students to improve their music training and performance skills. Using tools or plug-ins to assist in algorithmic composing can do some mode work with less effort. With the MIDI controller controlling the volume of the 8 tracks, you can control the various buttons and parameters on the transporter and the 4-band EQ.
\end{abstract}

\section{Keywords}

MIDI software $\bullet$ Ccontroller • Music Teaching • Simulated Annealing Algorithm

\footnotetext{
* This work was financially supported by Hunan social science fund project of Hunan province in 2016 (No. 16YBA061); university teaching reform research project of Hunan province in 2016 (Xiang Jiantong [2016] No. 400; Serial number: 644).

${ }^{1}$ Correspondence to: Jiaojing You (MA), School of Art, Hunan City University, Yiyang 413000, China. Email: youjj1981@163.com
}

Citation: You, J.J. (2018). Application of MIDI Software and Controller in Music Teaching. Educational Sciences: Theory \& Practice, 18, 1810-1818. http://dx.doi.org/10.12738/estp.2018.5.081 
Computer music is the principle of digital transmission using modern electronic computers. It adopts international MIDI technology standards and improves the electronic music system composed of asynchronous electronic equipment connected to several serial electronic instruments. The full name of MIDI is the digital interface of music equipment (Zhu, 2011; Wang, 2012; Wise, Greenwood \& Davis, 2011). It is the "Music Instrument Digital" in English. Abbreviation for Interface. For most people, the terms "computer music" and "MIDI production" are no strangers, but there are not many people who truly understand and master (Zhu, 2011; Nicholas, 2010). However, the birth and development of computer multimedia technology has had a tremendous impact on areas such as education, arts, entertainment, tourism, communications, and publishing, and has been deeply involved in all aspects of social life. The most important and popular of multimedia is computer music (Garner, Alley, Gaudelli \& Zappe, 2009). At present, computer music has swept the world and will become the mainstream of modern music. Most of the film music, television music, MTV music, and advertisement music use computer music to enhance the expressiveness of music works (Gelding, Thompson \& Johnson, 2015). And computer music is mostly made using MIDI technology. That is, through the MIDI interface, electronic musical instruments are connected with computers to form a computer music system. Using computer composing software, almost all sound effects can be produced, and computers can be directly used for composing and playing. Record and play, also print music scores. The computer music production system includes a software system and a hardware system (Guven, 2017).

\section{MIDI Music Production Software}

MIDI is the abbreviation of MIDI Digital Interface. It is literally translated as a digital interface for musical instruments. It is a universal protocol for the exchange of various digital musical instruments. MIDI music production software, that is, professional software and technology developed for music production based on MIDI technology. In MIDI music production, MIDI output is not the sound signal audio stream, but the control parameters, expressions and other instructions of the note. A MIDI system (software) is actually a system for composing music, orchestration, and electronic simulation (Abrizah \& Zainab, 2011).

\section{MIDI software work process}

Make use of tools or plug-ins to assist algorithmic composing. Using the Composing Tools in the plug-in, you can do some model work with less effort, such as Double Note Values, Halve Note Values, Find Motif, and Find Range. ), Invert, and Retrograde. Sometimes it is necessary to adjust the relationship between the sound and the sound. For example, if the major key is transformed into a minor or other special scale, you can use Pitch Mapping. It was also mentioned initially that the work can be done faster with the menu Notes/Add Interval or Add Pitch function (Simones, Schroeder \& Rodger, 2015). The process can see in the figure 1. 


\section{MIDI software assist algorithm}

I). You can store the motivation, pattern, or rhythm that you or others may use in the future. You can use Alt + I to call later: After you select an existing motivation, tone, or rhythm, copy and paste. In your new work.

II). You can use the smart copy and paste $(\mathrm{Ctrl}+\mathrm{Shift}+\mathrm{V})$ function to zoom in or out of the score in the score of the piano spectrum or the reduced spectrum according to your own idea or prefabricated style; or to change the existing score to the reduced spectrum.

III). Automatically generate percussion parts and select the desired style.

IV). Automatic harmony, optional column chord or decomposition chord, and harmonic transformation rhythm.

V). Automatic generation of Bass and Harmonic Skeleton

VI). Compress the multi-line spectrum into 1 spectrum.

VII). Change the scale mode, select various scales, and create your own pentatonic scale or other original scales. These two functions are interoperable.

VIII). Reflection, select the phrase, determine the axial sound $(\mathrm{C} 4=$ center $\mathrm{C})$ directly after the reflection.

IX). Retrograde, select the phrase to execute, it will be retrograde in another score, and you can copy it back to the original spectrum. This feature needs improvement.

$\mathrm{X})$. Music score analysis

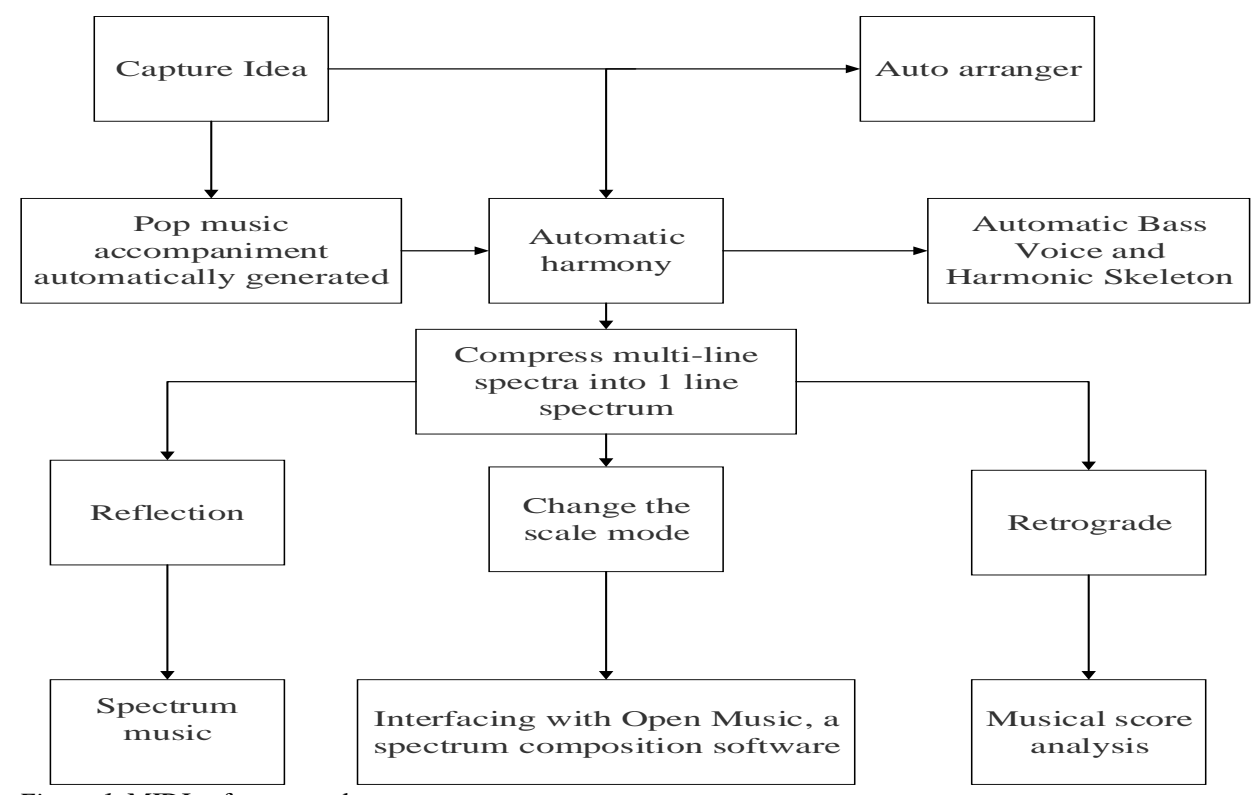

Figure 1. MIDI software work process. 


\section{Controller Tips and Features}

In the creation process of MIDI music, the use of the controller is a very important means. In order to create a higher level of modern music, the use of the controller is very important, and the use of the controller will make the original sound very rigid and It is not easy to hear that the music with strong sweetness has become humanized, and many of the details of music have been realized by it. The MIDI controller is 128 in international standards, and its various assignments are also 128 units. The digital information is represented as 0-127 instead of 1-128. Many people see that the maximum value that can be written in Cubase's MIDI control information is 127 , and the value of the misunderstanding control information is only 127 units. This is because of omission of the 0 result (Zhu, 2011). There are also some regular tutorials that will make GM sound libraries. Saying 127 timbres is caused by carelessness and lack of understanding of MIDI data. I hope that everyone will come out of this misunderstanding. Velocity information and pitch bend information are not part of the 128 MIDI controller information. The 128 controller information transmission data $\mathrm{BNH}$ is the position byte, and the velocity information is transmitted in the second data byte along with the note ON $9 \mathrm{NH}$. In addition, the pitch bend information has a value of 16384 units, the minimum is -8192 , and the maximum is 8191 , rather than the 0-127 that the control information should have. Take only the 8 knob controllers and 8 key controllers on the UMX61 keyboard as a demonstration.

Table 1

\begin{tabular}{cl}
\multicolumn{2}{c}{ Eight Knob Controllers Denoted by $R$} \\
\hline Knob Controller & Corresponding meaning \\
\hline R1 & Reverb Depth (CC91) \\
R2 & Chorus Depth (CC93) \\
R3 & Cut off Frequ (CC74) \\
R4 & Resonance (CC71) \\
R5 & Attack (CC73) \\
R6 & Decay (CC75) \\
R7 & Release (CC72) \\
\hline
\end{tabular}

Table 2

8 Button Controllers are Denoted by $B$

\begin{tabular}{cl}
\hline Key controller & Corresponding meaning \\
\hline B1 & Data Dec-1(CC97) \\
B2 & Data Inc+1(CC96) \\
B3 & Sostenuto(CC66) \\
B4 & Soft Pedal(CC67) \\
B5 & Sustain Pedal(CC64) \\
B6 & Porta On/Off(CC65) \\
B7 & Volume Mute(CC07) \\
B8 & Pan Centre (CC10) \\
\hline
\end{tabular}

\section{Music Teaching Effect Evaluation}

\section{Simulated annealing algorithm}

Simulated Annealing (SA) algorithm has a heuristic random search algorithm with global optimization, adaptive, fast convergence and so on (Zhu, 2011).. 
1) Initialization: initial temperature $T$ (fully large), initial solution states (that is, the iterative starting point of the algorithm), iteration number $\mathrm{L}$ of each $\mathrm{T}$ value, and control parameter $\mathrm{t}$.

2) For $\mathrm{k}=1, \ldots, \mathrm{L}$, repeat 3) 6).

3) Generate a new solution s'.

4) Calculate $\Delta \mathrm{f}=\mathrm{f}(\mathrm{s})^{\prime}-\mathrm{f}(\mathrm{s})$, where $\mathrm{f}(\cdot)$ is the evaluation function.

5) If $\Delta \mathrm{f}<0$, then accept $\mathrm{s}^{\prime}$ as a new solution; otherwise, accept $\mathrm{s}^{\prime}$ as a new solution with probability e $(-\Delta \mathrm{f} / \mathrm{T})$.

6) If the termination condition is satisfied, the current solution is output as the optimal solution and the program exits. Wherein, the termination condition is generally taken to be when several consecutive new solutions have not been accepted.

7) T gradually decreases and tends to 0.8 ) Turn 2).

The process can be seen in figure 2 .

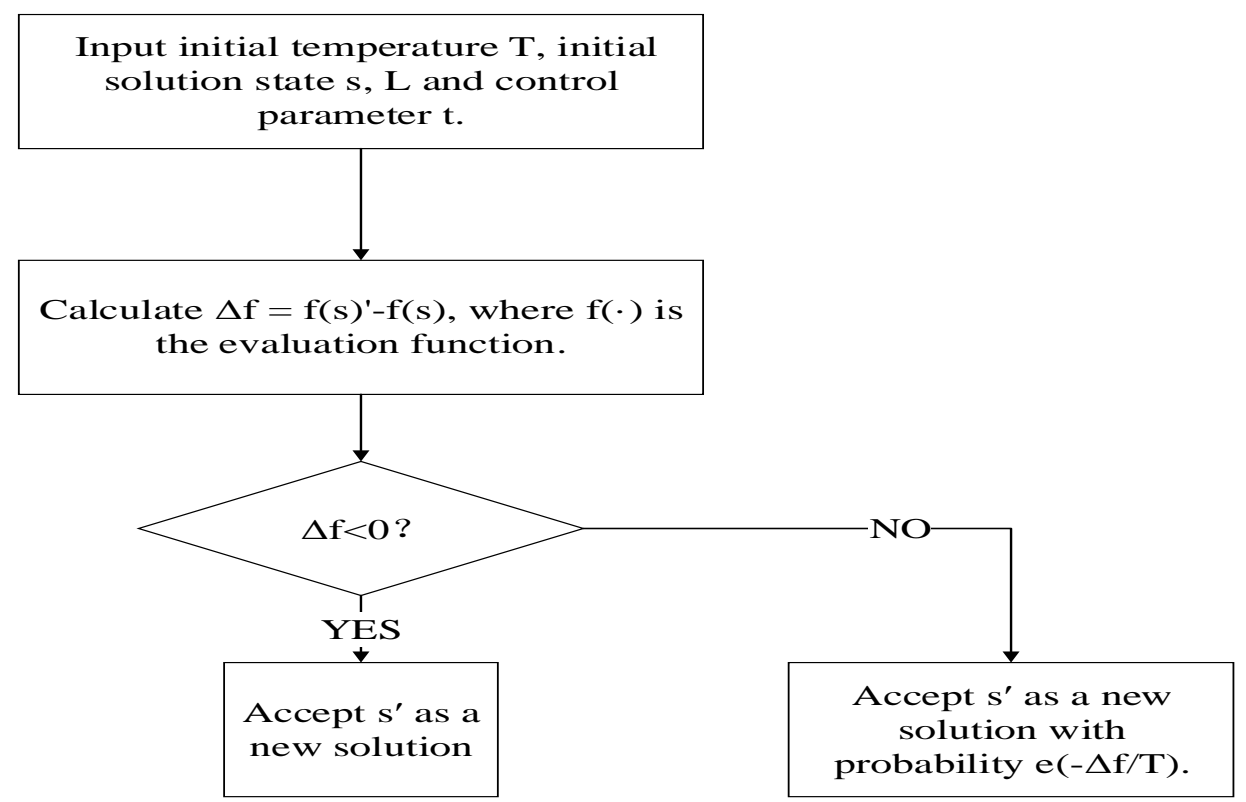

Figure 2. Simulated annealing algorithm.

\section{SA-based music teaching evaluation algorithm}

In order to make an objective and quantitative assessment of the player's performance results, not only must a scientific overall evaluation of the performance be made, but also every keystroke of the performer needs to be evaluated, including each Whether the button is right or wrong, the timing of the button, the length, whether the button is pressed, and the button is pressed. To this end, the results of music masters can be used as a 
standard for comparative evaluation.[3] In order to reduce the complexity of the algorithm design, the judgment of chords is not considered. In order to use the advantage of the simulated annealing algorithm to find the global optimal solution and the fast convergence speed, the standard track is divided into several key groups according to the score spectrum, and the following evaluation functions are introduced for each group of keys:

$$
f=\sum\left(x-1 \cdot \frac{T_{\text {real }}-T_{\text {std }}}{T_{\text {std }}}\right)+\sum \frac{T_{\text {real }}^{\prime}}{\operatorname{avg}\left(T_{\text {std }}\right)} / N
$$

where $\mathrm{x} \in\{0,1\}$, and $\mathrm{x}=1$ indicates that the button is correct; 0 indicates a button error or fewer buttons, $\mathrm{T}_{\text {real }}$ is the actual button duration, $\mathrm{T}_{\text {std }}$ is the length of the standard button, $\mathrm{T}_{\text {real }}$ is the length of the multiple button, and $\operatorname{avg}\left(\mathrm{T}_{\text {std }}\right)$ is the average standard The length of the key, $\mathrm{N}$ is the number of keys. Then for the entire performance (and all the actual keys) there is the following SA evaluation:

$$
F=\min \sum f
$$

When applying the simulated annealing algorithm to generate a new solution, the constraints that should be satisfied are as follows:

$$
\begin{aligned}
& \alpha 1=\sum_{T_{\text {real }}} \in o(1, \delta) \\
& \alpha 2=\frac{\sum T_{\text {std }} \text { standard number of keys }}{\sum T_{\text {real }} \text { actual number of keys }} \in o(1, \delta)
\end{aligned}
$$

\section{The algorithm for judging whether to press the wrong button, press the multi-button, less buttons}

For the convenience of judgment, the introduction of the following concepts:

The ratio of the primary key to the standard key length

$\rho=\sum_{\sum T_{\text {real }}}^{\sum T_{\text {std }}} \in o(1, \delta)$, where $\delta$ is a normal amount of $<1$.

Minor key: The ratio of the length of the key to the standard key $\rho<1-\delta$.

The wrong button: is the wrong button.

I). How to determine whether the actual key is the primary key

Without loss of generality, it is assumed that i-1 keys before the i-th actual key have been judged, and now it is judged from the i-th key whether the actual key is the primary key (Nicholas, 2010).

When $\rho \geq 1-\delta$, regardless of whether the type of button is correct, we all think that the button is wrong, and no longer consider it; 
When $\rho \in \mathrm{o}(1, \delta)$, this button is the primary key. The primary key will enter the next round, waiting for further evaluation;

When $\rho<1-\delta$,

$\alpha>1+\delta$, Situation 1

$\alpha \in \mathrm{o}(1, \delta), \beta=\frac{\sum T_{\text {real }}}{\sum T_{\text {std }}}>1$ Situation 2

$\beta=\frac{\sum T_{\text {real }}}{\sum T_{\text {std }}}<1$ Situation 3

$\alpha<1-\delta, \beta \beta=\frac{\sum T_{\text {real }}}{\sum T_{\text {std }}}>1$ Situation 4

$\beta=\frac{\sum T_{\text {real }}<1}{\sum T_{\text {std }}}<1$ Situation 5

Where $\alpha=\frac{T_{\text {real }}(i+1)}{T_{\text {real }}(i)}$.

Case 1 is the i-th key as the primary key; Case 2 is the i-th or i+1-th key that is the primary key, which will be judged based on the specific key condition; Case 3 is the $i$-th and $i+1$-th key is the secondary key, $i+2$ keys are primary keys; case 4 is $i, i+1$, and $i+2$ keys are secondary keys, $i+3$ keys are primary keys; case $5 i$ to $i+3$ keys are secondary keys, and no The primary key corresponding to this standard key (Nicholas, 2010).

II). The algorithm for judging the key condition is such that Treal(start) is the start time of the i-th actual keystroke, Tstd(end) is the time when the i-th criterion key ends, and $\delta$ is a certain normal amount. Introduce the following judgment conditions:

$$
T_{\text {real }}(\text { start })-T_{\text {std }}(\text { end })>-\delta
$$

Then the algorithm can be simply described as:

(1) Perform the following operations for each group of buttons.

(2) $\mathrm{i}=1, \ldots, \mathrm{L}, \mathrm{L}$ is the number of keys in the group, then the $\mathrm{i}$-th key is:

(a) To determine whether formula (4) is satisfied;

(b) If satisfied, go to (d);

(c) Otherwise, the evaluator leaks the key;

(d) Under the condition of satisfying formula (3), find the primary key according to the above-mentioned algorithm;

(e) If a primary key is found, compare it with a standard key, and if it is the same, the key is correct; if it is not the same, the key is incorrect; 
(f) Otherwise the examined keys are secondary keys, which are multi-pressed keys.

(3) Output the result and jump out.

The algorithm flow is shown in Figure 3.

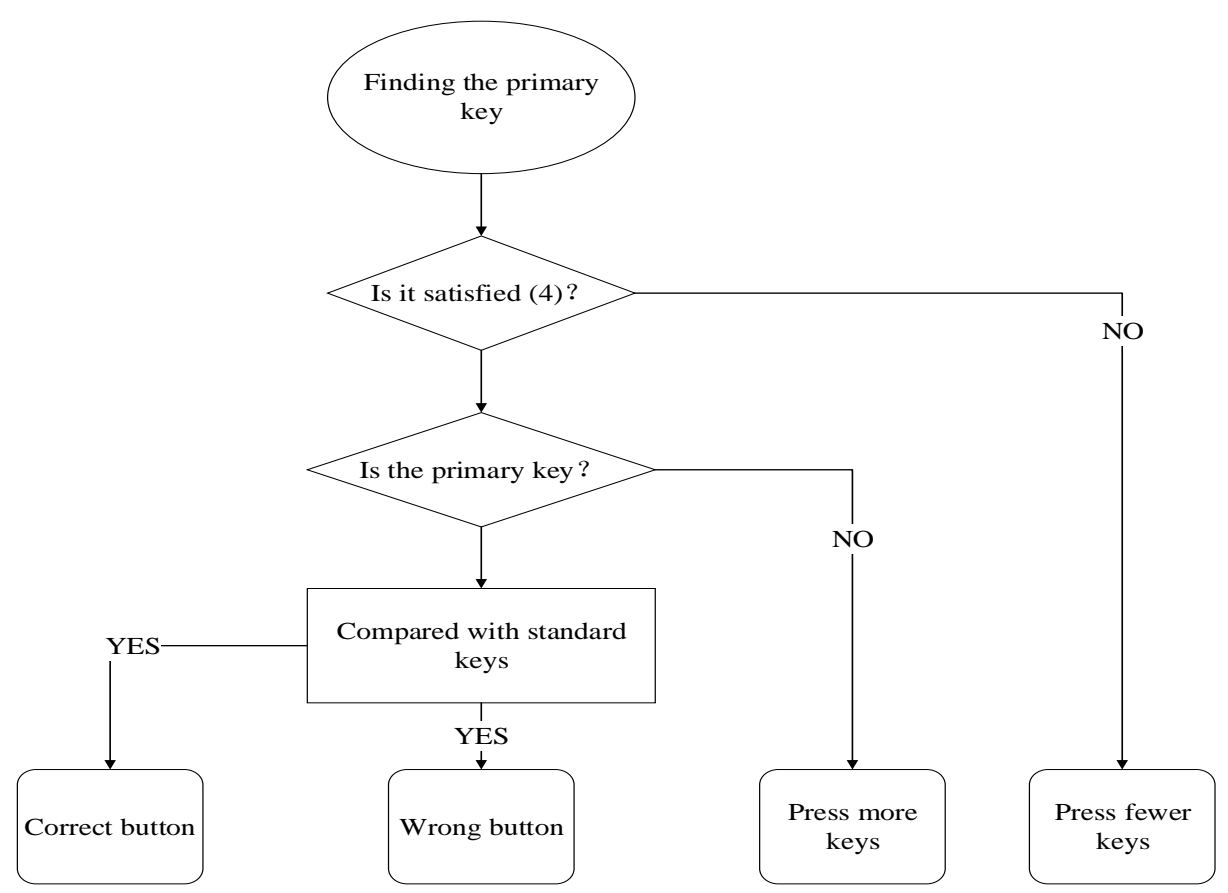

Figure 3. Algorithm flow for judging button conditions.

With the above discussion, a simulated annealing algorithm can be used to make a scientific and objective assessment of performance results. In addition to the overall evaluation $\mathrm{F}^{*}$ of the performance of the appraiser, and the reference to the construction of the optimal solution of the SA algorithm, the algorithm can be used to infer the right and wrong of each button, the timing, the length, the keystrokes, whether the button is pressed, the buttons are less, etc. This will guide the appraisers to improve their playing skills (Nicholas, 2010).

\section{Conclusion}

In short, the introduction of modern technology means to improve teaching quality is the specific implementation of the requirements for education innovation in the new era. The practical application of MIDI technology and computer music production system in training is effective in solving technical problems in music training. At the same time, it also improved students' deeper understanding of music and enabled them to have more exposure to rich timbres, various rhythm styles, and different times and harmony styles in the 
process of listening, learning, singing and watching. Cultivate its ability to perceive music, participate in the ensemble and concerto performance of large band performances, shorten the distance between students and music, truly provide technology and means for education services, serve music, and make teaching content more Expanding space will surely become one of the modern teaching methods that modern music teachers must master. It is believed that the future of China's music education and music arts will be brighter and brighter. It will shine in the most dazzling colors in social development.

\section{References}

Abrizah, A., \& Zainab, A. N. (2011). Digital libraries in the classroom: secondary school teachers' conception. Journal of Librarianship \& Information Science, 43(4), 224-236. https://www.jstor.org/ 10.1177/0961000611418811

Garner, J.K., Alley, M., Gaudelli, A.F., \& Zappe, S.E. (2009). Common use of power point versus the assertionevidence structure: a cognitive psychology perspective. Technical Communication, 56(4), 331-45, https://www.jstor.org/stable/43094155.

Gelding, R. W., Thompson, W. F., \& Johnson, B. W. (2015). The pitch imagery arrow task: effects of musical training, vividness, and mental control. PloS One, 10(3), 1-10. http://dx.doi.org/10.1371/journal.pone.0121809

Guven, E. (2017). Levels of music performance anxiety and test anxiety of Turkish prospective music teachers in piano exams. International Journal of Music Education, 35(2), 154-164. https://www.jstor.org/ $10.1177 / 0255761415620530$

Nicholas, R. (2010). Technology and computers in music and music education. key competencies in the knowledge society. Springer Berlin Heidelberg, 333-343. http://dx.doi.org/10.1007/978-3-642-15378-5_32

Simones, L., Schroeder, F., \& Rodger, M. (2015). Categorizations of physical gesture in piano teaching: A $\begin{array}{llll}\text { preliminary enquiry. Psychology of } & \text { Music, }\end{array}$ https://www.jstor.org/10.1177/0305735613498918

Wang, X. (2012). Multimedia Systems in Music Teaching of Normal University. Procedia Environmental Sciences 12. B, 1248-1252. http://dx.doi.org/10.1016/j.proenv.2012.01.416

Wise, S., Greenwood, J., \& Davis, N. (2011). Teachers' use of digital technology in secondary music education: illustrations of changing classrooms. British Journal of Music Education, 28(2), 117-134. http://dx.doi.org/10.1017/S0265051711000039

Zhu, J. (2011). MIDI and music teaching in colleges of multimedia system application. Key Engineering Materials, 474-476, 1926-1930. http://dx.doi.org/10.4028/www.scientific.net/KEM.474-476.1926

Zhu, J. (2011). Multimedia music teaching system application. Key Engineering Materials, 474-476, 19031908. http://dx.doi.org/10.4028/www.scientific.net/KEM.474-476.1903 